at a health clinic and were divided into quintiles according to BMI. During a mean follow-up of $16.7 \pm 7.8$ years, 592 women developed hypertension. According to multivariate regression analysis, increased baseline BMI was significantly associated with an increased risk of developing hypertension, even within the normal BMI range $\left(P_{\text {trend }}<0.001\right)$. Relative to women with a BMI of $18.5-20.0 \mathrm{~kg} / \mathrm{m}^{2}$, those with a BMI of $20.1-21.2 \mathrm{~kg} / \mathrm{m}^{2}$ had a hazard ratio for hypertension of 1.16 . The association between BMI and hypertension was not seen in women $\geq 55$ years old and was independent of cardiorespiratory fitness. Percent body fat and fat mass were also significantly and positively associated with increased incidence of hypertension ( $P_{\text {trend }}<0.05$ for both), but no such association was seen for waist circumference or fat-free mass.

The authors conclude that future studies that include individuals who are more representative of the general female population need to be conducted before the association between $\mathrm{BMI}$ and hypertension can be confirmed.

Original article Shuger SL et al. (2008) Body mass index as a predictor of hypertension incidence among initially healthy normotensive women. Am J Hypertens 21: 613-619

\section{New study contradicts suggestions of an 'epidemic' of primary hyperaldosteronism}

Studies conducted in tertiary referral centers over the past 8 years reported a controversially high prevalence of primary hyperaldosteronism in patients with resistant hypertension (14-23\%). Historically, the prevalence of this condition was around $1 \%$, and this discrepancy led some researchers to claim that primary hyperaldosteronism has become an epidemic. Doumas and colleagues from Thessaloniki, Greece, have published their 20-year experience of patients with resistant hypertension, which most authorities agree is the factor most strongly associated with primary hyperaldosteronism. Their results suggest that referral and selection bias have probably inflated these estimates of hyperaldosteronism's prevalence.

This retrospective, observational study included 1,616 patients with resistant hypertension despite full doses of a diuretic and two antihypertensive medications. After a washout period off diuretics and $\beta$-blockers, measurement of serum aldosterone and plasma renin levels identified 338 (20.9\%) patients with aldosterone levels $>416 \mathrm{pmol} / \mathrm{l}$ and an aldosterone:renin ratio $>65.16$. Primary hyperaldosteronism was confirmed in 182 of 1,616 (11.3\%) patients by $4 \mathrm{~h}$ intravenous salt loading, 4 days of fludrocortisone administration and a response to spironolactone.

Doumas and colleagues observed hypokalemia in significantly more patients with primary hyperaldosteronism than without it (45.6\% vs $15.9 \%$ ), although this finding could not confirm the diagnosis. They highlight that screening for primary hyperaldosteronism by aldosterone:renin ratio and aldosterone levels generated large numbers of false-positive results; however, fludrocortisone administration was the gold-standard diagnostic test for primary hyperaldosteronism, since it correlated with patients' responses to spironolactone.

Original article Douma S et al. (2008) Prevalence of primary hyperaldosteronism in resistant hypertension: a retrospective observational study. Lancet 371: 1921-1926

\section{Orally administered tetrahydrobiopterin can reduce hypertension}

Tetrahydrobiopterin is a cofactor in the formation of nitric oxide, and hypertension has been shown to inhibit the function of tetrahydrobiopterin, leading to a decrease in nitric oxide bioavailability. Porkert et al. conducted two pilot studies that investigated the effect of orally administered tetrahydrobiopterin on blood pressure (BP) and the dose-response relationship of this effect.

Both pilot studies enrolled patients (aged 18-75 years) who had uncontrolled hypertension $(\geq 135 / 85 \mathrm{mmHg}$ ) while on conventional medication or newly diagnosed hypertension $(\geq 140 / 90 \mathrm{mmHg})$. The first study included 8 patients who were assigned to receive either $2.5 \mathrm{mg} / \mathrm{kg}$ or $5 \mathrm{mg} / \mathrm{kg}$ of tetrahydrobiopterin twice daily for 8 weeks, and the second study included 16 patients who were allocated to receive either $100 \mathrm{mg}$ or $200 \mathrm{mg}$ of tetrahydrobiopterin twice daily for 4 weeks. All patients continued their pre-existing antihypertensive therapy. Both groups in the first study and the $400 \mathrm{mg} /$ day group in the second 